\title{
Three-dimensional mobile mapping system and its use in road engineering
}

\author{
Zuzana Florkova ${ }^{1, *}$, Lukas Duris ${ }^{1}$, Michal Veselovsky ${ }^{1}$, Stefan Sedivý ${ }^{2}$, and Dasa \\ Kovalova $^{1}$ \\ ${ }^{1}$ University of Zilina, Research Centre, Univerzitna 8215/1, Zilina SK- 010 26, Slovak Republic \\ ${ }^{2}$ University of Zilina, Department of Construction Management, Univerzitna 8215/1, Zilina SK- 010 \\ 26, Slovak Republic
}

\begin{abstract}
The paper focuses on the issue of the use of three-dimensional mobile mapping system and the following processing of obtained data. The first part is devoted to the description of the three-dimensional mobile mapping technology using LiDAR, specifically to the mobile threedimensional scanner - Lynx SG1 from Teledyne OPTECH. It describes into more details the process of works from the field data collection to their so called "postprocessing" as well as a variety of output options and interpretations of results obtained in the measurements. Advantages of the system together with its limits of use are summarized in the conclusion of the research paper.
\end{abstract}

\section{Introduction}

The fast development and advancement of new technologies have brought great changes to geodesy and mapping as well. The quick implementation of geographic information systems (GIS) and their never ending need for accurate and current spatial data promotes the development of new approaches for automated and fast data acquisition [1]. The number and complexity of the current civil engineering infrastructures makes it necessary to use accurate, fast and reliable monitoring systems to ensure the safety both during construction and service life [2]. Increasingly are used photogrammetry or scanner technologies - static scanners, mobile mapping systems, airborne laser scanning. Their main advantage is the short period of time needed for data collection compared to conventional methods, which is used to map the territory, buildings or communications where it is necessary to avoid any or, respectively minimum restrictions during data collection.

Mobile laser scanning is a new and rapid system to capture high-density threedimensional point cloud. Automatic data segmentation and feature extraction are the key steps for accurate identification and three-dimensional reconstruction of street-scene objects (e.g. roads, buildings, trees etc.) [3].

This paper deals with the description of the mobile mapping system (Lynx SG1 from Teledyne OPTECH) and also describes in more detail the processing method of obtained data which were measured on a particular section of the road and its surroundings. The

\footnotetext{
* Corresponding author: zuzana.florkova@,rc.uniza.sk
} 
communication in the residential area was chosen because of diversity of scanning elements located in this area, as well as the good "range" of the GNSS antennas.

\section{Mobile mapping system}

Mobile mapping system (MMS) allows a contactless determination of spatial coordinates of the points with a moving vehicle, sensors and software that is able to evaluate the collected data. Mobile laser scanner is able to collect a huge amount of points with a minimum pitch in a very short time - also known as point cloud. At the same time as digital cameras and created images of scanned objects with which it is possible to every point in cloud assign color according to its true colors. The result may therefore be a point cloud with high density or three-dimensional model in real colors placed in the desired coordinate system [4]. Integrated in a mobile mapping system, the data acquired by laser scanners can be used to robustly capture the geometry of the road surrounding and be the basis for the recognition of a wide range of objects. A detailed classification of objects, in particular traffic signs and road lanes will, however, remain largely based on optical imagery [5].

Mobile mapping process generally consists of the data collection (using a mobile mapping device), postprocessing (processing of measured data) and point cloud processing.

For the purpose of mobile mapping device combines a several technologies at a time together (light detection and ranging device (LiDAR), global navigation satellite system (GNSS), inertial measurement units (IMU), vehicle distance measurer (DMI)).

Light detection and ranging (LiDAR) a method that measures distance to a target by illuminating that target with a pulsed laser light and measuring the reflected pulses with a sensor. Differences in laser return times and wavelengths can then be used to make digital representations of the target [6].

Global navigation satellite system (GNSS) - real-time positioning of LiDAR - allows to place the resulting point cloud in the required coordinate system with the required precision.

Inertial measurement unit (IMU) - detects dynamic vehicle parameters - integrates gyroscopes, accelerometers, and vehicle distance measurer (DMI). By connecting them, GNSS can overcome satellite signal failure and also determine the orientation of the scanners in space.

Vehicle distance measurer (DMI) - Vehicle distance and speed measurement - to improve the accuracy of GNNS and IMU. Especially in sections with weak GNNS signals.

The raw data from the measurement by MMS are data obtained from observational GNSS antenna mounted on the vehicle, from the basic (static) station GNSS, data from the IMU unit, photographs taken from digital cameras and point clouds. These data are used as a main data input for the postprocessig (additional processing and refinement of the obtained data). The result of postprocessig is a point cloud located in the selected coordinate system, where the each point includes information about color, position, height and intensity. Depending on further processing it is possible to export these data in various formats (*.las, *.asc, *.kml, *.shp, etc.). This processing involves the classification of the point clouds according to the selected criteria (reflection intensity, point color, altitude etc.). It is an important process in which it is possible to use the appropriate tools for the purpose of separation of the individual elements of the mapped area. For example, vegetation, trees, buildings, power lines, etc. Depending on the required results from mobile mapping, it is possible also vectorize the point clouds (terrain edges, buildings) together by modeling individual objects, or to perform another analyzes in different GIS applications. 


\section{Mapping using the Lynx SG1 mobile system from Teledyne OPTECH}

The following part of the contribution is devoted to the measurement principle and to a more detailed description of the data processing procedure by the Lynx SG1 mobile mapping device (Figure 1) owned by University of Zilina Research Center.

The working process of mobile mapping is based on the following steps:

1. Data collection using a mobile laser unit - Lynx SG1 and digital cameras including $360^{\circ}$ Spherical cameras - Ladybug,

2. Vehicle trajectory refinement - using software POSPac 7.2,

3. Combine calculated vehicle trajectory with data from laser unit and cameras and then export to *.las file. - using the LMS 4.1 software,

4. Processing *.las file - classifying individual groups of points,

5 . Processing *.las file - vectoring of point cloud + export *.dwg or *.dgn.

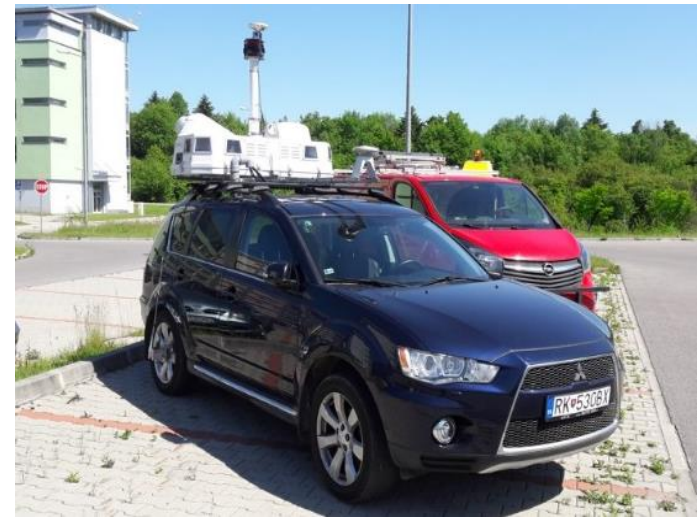

Fig. 1. Mobile mapping vehicle Lynx SG1 owned by University of Žilina Research Center

\subsection{Data collection}

Data collection was carried out in urban in the area of a predominantly individual residential development (Figure 2). The area of the mapping was selected due to the variety of terrain element (buildings, street lighting, trees, bushes, paved and unpaved surfaces) as well as GPS signal quality.

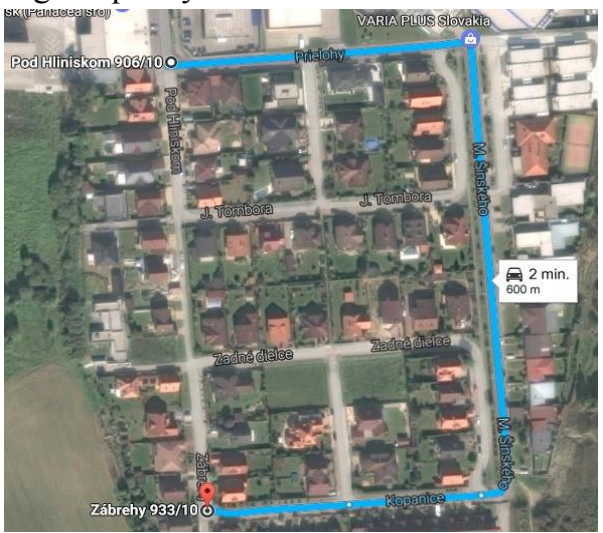

Fig. 2. Data collection area. 


\subsection{Calculation of vehicle trajectory}

The position data were used to calculate of the vehicle trajectory. These data was obtained by GNSS antennas mounted on mobile mapper vehicle and by static (permanent) base stations GNSS. Data conditioning was carried out by POSPac software (version 7.1) supplied by Applanix. Permanent station located on the roof of the University of Žilina, which record data at 15 seconds interval was used as the static base. Both, data collection and processing in POSPac were performed in the European Terrestrial Reference System 1989 (ETRS89). Then, the result of this process is smoothed best estimate of trajectory (SBET).

\subsection{Point cloud calculation}

The result output format was a point cloud where each point includes information about the intensity of the reflection, colour and position in the selected reference frame. Depending on the further use of point cloud it can be displayed in various ways, for example according to the intensity of the reflected beam (Figure 3 left), in real colours (Figure 3 right) or altitude (Figure 4).

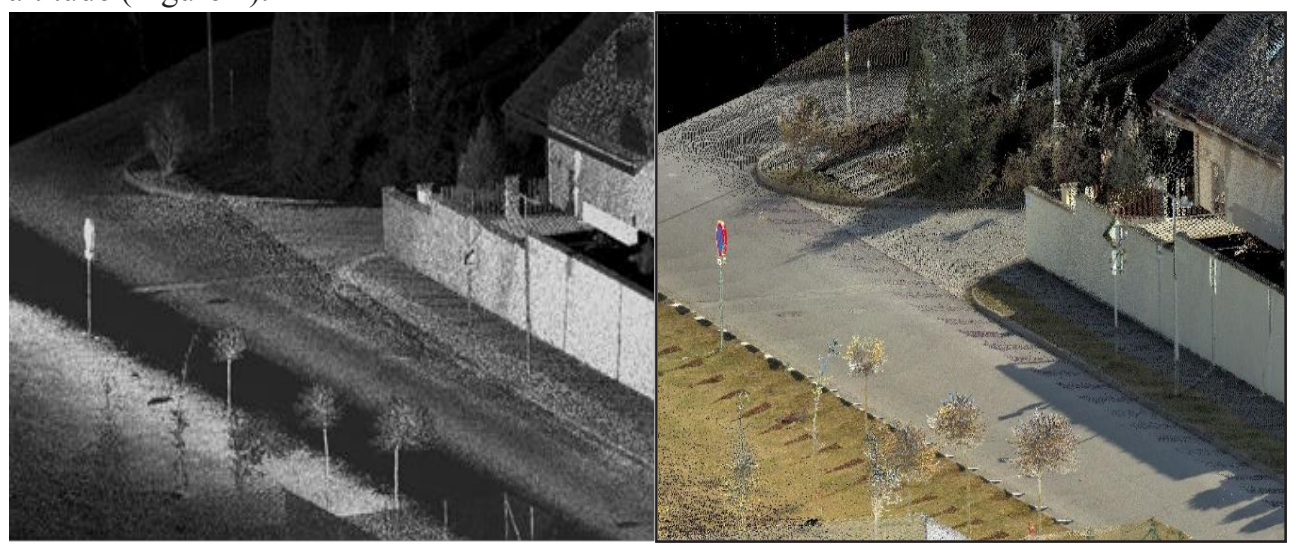

Fig. 3. Left: standard view of point cloud. Right: view of point cloud in real colours

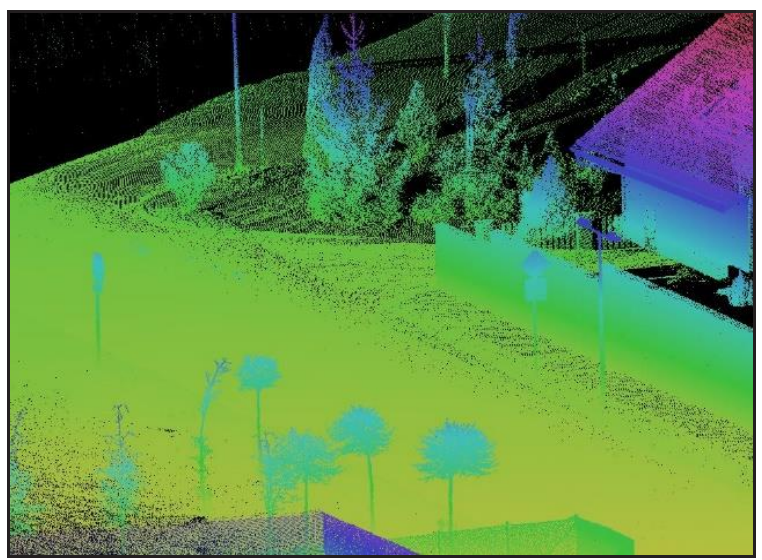

Fig. 4. View of point cloud according to the altitude

The software provided by Teledyne OPTECH - LMS 4.1 was used to calculate the point cloud. Input data for the point cloud calculation were: files with the accurate trajectory of 
the vehicle - SBET, photos from digital cameras and $360^{\circ}$ LADYBUG cameras, and LiDAR data. The ETRS89 reference framework was selected for the final data processing in the LMS software. Then the point cloud is the resulting output format and each point of this format include the information about the reflection intensity, colour and the position in the selected reference framework

\subsection{Point cloud processing}

The Terrascan software from Terrasolid company, was used to process point cloud in * .las format. Software Terrascan works as an extension for MicroStation V8i and offers a wide range of tools and options for working with point cloud. In the first step, in order to obtain the clearest rendering, it is necessary to classify points by separating individual objects (e.g. sidewalks, pavements, grassy areas etc.). In the second step it is important to remove unnecessarily objects (e.g. cars, faulty objects). Classification of points can be performed automatically, semi-automatically and manually. Points can be filtered by colour, intensity of reflected signal, altitude, and other criteria, and link them to groups with common attributes. A good point cloud classification is the base for further analysis and work. Figure 5 shows the classification of points by the following groups: pavement (orange color), low green (dark green), trees, bushes (light green) and elements defined for removing. - cars (red colour).

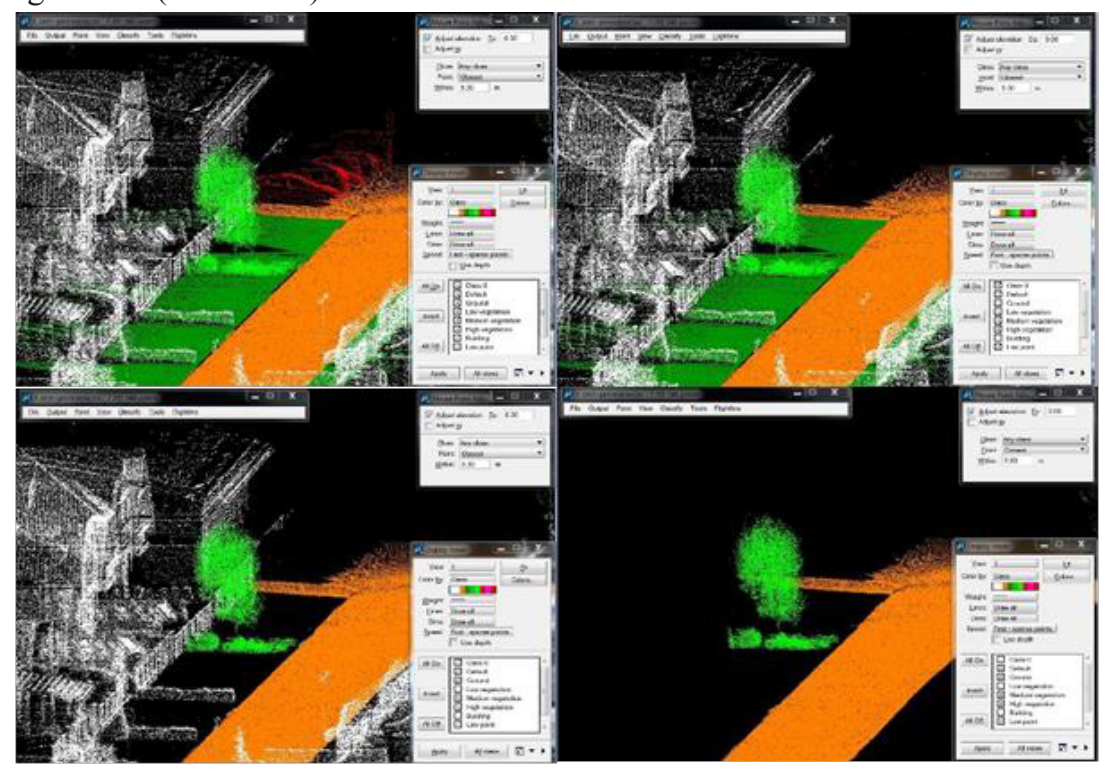

Fig. 5. Classification of points in Terrascan

\subsection{Vectorization}

There are several tools in the Terrascan software extension to vectorize the edges and elements from the point cloud. Tool "Mouse point adjustment" is important and allows to set mouse clicks to individual objects, elements, point classes, etc. This tool allows clear and correct identification and plotting of the required lines and objects (Figure 6). It is possible to export of point cloud output to various standard formats useful for designers, for example *.dgn, *.dwg, *.shp, after vectorization process. 
In this case, all data, including vectorization, are processed in the ETRS89 coordinate system. Finally, a *.dgn drawing is created, which is transformed by transformation service into the SJTSK (Single Cadastral Trigonometric Network System). This transformation allows the location of measured into the Slovak Republic area. Subsequently it is possible to work with these data also in the field of real estate cadastre (Figure 7).

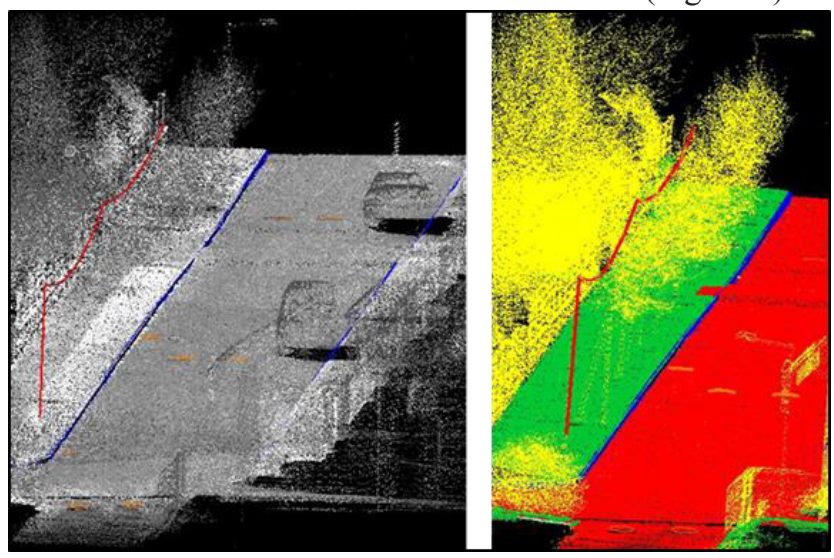

Fig. 6. Point cloud vectorization

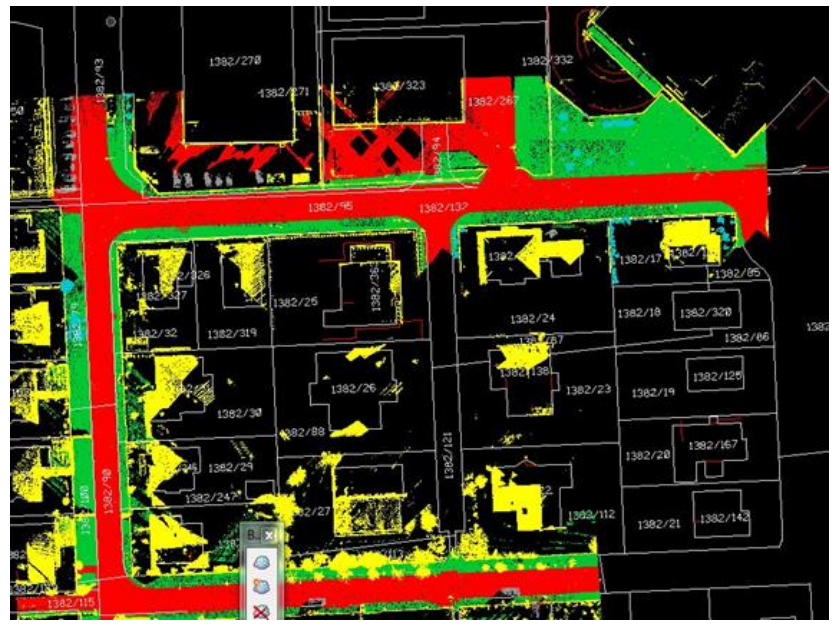

Fig. 7. Location of the mapped are in the cadastral map

\section{Conclusion}

The paper presents mobile mapping technology by LiDAR device with using the Lynx SG1 three-dimensional scanner on a concrete example of scanning of road communication and its surrounding. In short order, it presents the process of data acquisition, data processing, output options and their interpretation. Presented processed outputs can then be used in various fields of road engineering, whether in the design phase, the construction phase or in the subsequent updates of the state of road construction during their long-term use. Obtained output allows designers to work directly in a three-dimensional digital terrain model, and thus better to design and to situate of construction.

Application of this mobile mapping system not only in the field of road engineering, brings undoubtedly many advantages, mainly including the speed of scanning and accuracy 
of collected data. The advantage of this system is that if the three-dimensional model of scanned area is not required in real colours, it is possible to collect data regardless of lighting conditions.

However, this three-dimensional scanning technology has also disadvantages and limitations. It is mainly the low quality of the GNSS signal reception, especially in densely built-up areas and in areas with high vegetation. These areas blocking or reducing the quality of the GNSS signal reception and thereby the absolute accuracy of the measurement is decreasing. The reduced quality or drop-out of GNSS signal reception can be replaced by addition of manually collected geodetic points in the post-processing phase. The lower is the quality of the signal, then more points are need to be added to achieve the maximum possible accuracy. Another limit for the usage of three-dimensional scanning are factors such as rain, fog or shiny surfaces because of reducing the reflectivity of LiDAR beams. Measurements presented in this paper were carried out without usage of geodetic points and the absolute accuracy of position was achieved less than $3 \mathrm{~cm}$. In this case, it is possible moreover to improve accuracy with usage of additional geodetic points.

This research was supported by European project Research Centre of the University of Žilina Second Phase: ITMS 313011D011.

\section{References}

1. K. Novak, Mobile mapping technology for GIS data collection. Photogramm. Eng. Remote Sensing, 61, 5, 493-500, (1995)

2. H.Gonzalez-Jorge, et al., Novel method to determine laser scanner accuracy for applications in civil engineering. Opt. Apll., 42, 1, 43-53, (2012)

3. B. Yang, et al., Automated extraction of street-scene objects from mobile lidar point clouds. Int. J. Remote Sens, 33, 18, 5839-5861, (2012)

4. C. V. Tao, Advances in mobile mapping technology: Volume 4 of International Society for Photogrammetry and Remote Sensing book series. (Taylor \& Francis. ISBN 0-41542723-1, 2007)

5. S. Pu, et al., Recognizing basic structures from mobile laser scanning data for road inventory studies. ISPRS J. Photogramm. Remote Sens., 66, 6, (2011)

6. NOAA, What is LIDAR? National Ocean Service website, https:/oceanservice.noaa. gov/facts/lidar.html 